\title{
In Situ Hybridization Shared Resource
}

National Cancer Institute

\section{Source}

National Cancer Institute. In Situ Hybridization Shared Resource. NCI Thesaurus. Code C39426.

The In Situ Hybridization Shared Resource provides Cancer Center investigators with histological review and interpretation of immunohistochemical and in situ hybridization studies of human and experimental animal solid tumor specimens. 